\title{
Reconstrução combinada do ligamento cruzado Anterior e lesão do ligamento anterolateral comparada àreconstrução isolada do ligamento cruzado anterior: Uma metanálise
}

\section{Combined Reconstruction of the Anterior Cruciate Ligament and Anterolateral Ligament Injury Compared with Isolated Reconstruction of the Anterior Cruciate Ligament: A Meta-Analysis}

\author{
Augusto Leão Bucar ${ }^{1}$ Rodrigo Nunes de Albuquerque Pires ${ }^{1}$ Rodrigo do Carmo Silva ${ }^{1}$ \\ Edicarlos André Cavalcante de Araujo ${ }^{1}$ Messias Froes da Silva ${ }^{1}$ Pedro Henrique Nunes de Araujo ${ }^{1}$ \\ ${ }^{1}$ Departamento de Ortopedia, Hospital das Forças Armadas, Brasília, \\ Endereço para correspondência Augusto Leão Bucar, MD, Hospital \\ DF, Brasil \\ das Forças Armadas, SQNW, 310, bloco F, apartamento 509, Brasília, \\ DF, 70675-731, Brasil (e-mail: augustobucar@hotmail.com).
}

Rev Bras Ortop 2021;56(1):24-30.

\begin{abstract}
Resumo
O objetivo da presentepesquisa é comparar, por meio de uma metanálise, os resultados da reconstrução combinada do ligamento cruzado anterior (LCA) e do ligamento anterolateral (LLA), comparado com a reconstrução isolada padrão, em pacientes com lesão crônica do ligamento cruzado anterior. Buscando alcançar o objetivo da pesquisa, foi realizada uma meta-análise para determinar se a combinação da reconstrução combinada LCA e LLA levaria àmelhoria significativa da função do joelho, medida pelos escores de avaliação International Knee Documentation Committee (IKDC), Lysholm, KT-2000 e menor taxa de ruptura do enxerto, em comparação com a reconstrução isolada. Para identificar ensaios clínicos randomizados (ECR) comparando a reconstrução combinada do LCA e LLA com a reconstrução isolada do LCA, foram pesquisados artigos publicados entre 2010 e 2019 nas

Palavras-chave

- ligamento cruzado anterior

- ligamento anterolateral

- lesão ligamentar

- ruptura

- lesão crônica bases MEDLINE, EMBASE, SPORTDiscus, LILACS e Cochrane Central RegisterofControlledTrials e seguiram os critérios de Itens de Relatórios Preferidos para Revisões Sistemáticas e Metanálises (PRISMA). A estabilidade da articulação do joelho é apenas marginalmente aprimorada com a reconstrução combinada de LCA e LLA, e ambas as técnicas de reconstrução mostram resultados funcionais. Os principais desfechos procurados foram a função do paciente e as taxas de estabilidade e ruptura do enxerto após a reconstrução do LCA. Dos 421 estudos identificados, 6estudos foram incluídos em nossa meta-análise. A qualidade do estudo (validade interna) foi avaliada usando o instrumento Cochrane risco-
\end{abstract}

Trabalho desenvolvido no Hospital das Forças Armadas, SQNW,

310, bloco F, apartamento 509, Brasília, DF, Brasil.

recebido

16 de Setembro de 2019

aceito

02 de Março de 2020

Publicado online

Novembro 2, 2020
DOI https://doi.org/ 10.1055/s-0040-1709990. ISSN 0102-3616.
(C) 2020. Sociedade Brasileira de Ortopedia e Traumatologia. All rights reserved.

This is an open access article published by Thieme under the terms of the Creative Commons Attribution-NonDerivative-NonCommercial-License, permitting copying and reproduction so long as the original work is given appropriate credit. Contents may not be used for commercial purposes, or adapted, remixed, transformed or built upon. (https://creativecommons.org/ licenses/by-nc-nd/4.0/)

Thieme Revinter Publicações Ltda., Rua do Matoso 170, Rio de Janeiro, RJ, CEP 20270-135, Brazil 


\section{Abstract \\ Keywords \\ - anterior cruciate ligament \\ - anterolateral ligament \\ - ligament injury \\ - rupture \\ - chronic lesion}

de-viés; em geral, foi encontrada uma qualidade moderada de evidências dos estudos incluídos. Os pacientes submetidos à reconstrução isolada do LCA mostraram maior taxa de ruptura do enxerto (RR 0,22; índice de confiança [IC]95\%: 0,12-0,41; $p<0,00001$ ). The present research aims to compare the outcomes from the combined reconstruction of the anterior cruciate ligament (ACL) and of the anterolateral ligament (ALL) with the standard isolated $A C L$ reconstruction in patients with chronic $A C L$ injury. To do so, a meta-analysis was carried out to determine whether the combined $A C L$ and ALL reconstruction would lead to a significant improvement in knee function according to the International Knee Documentation Committee (IKDC), the Lysholm test and KT2000 evaluation scores and lower graft rupture rates in comparison with isolated reconstruction. To identify randomized controlled trials (RCTs) comparing the combined $A C L$ and $A L L$ reconstruction with the isolated $A C L$ reconstruction, papers published between 2010 and 2019 were searched in the MEDLINE, EMBASE, SPORTDiscus, LILACS and Cochrane Central Register of Controlled Trials databases, following the Preferred Reporting Items for Systematic Reviews and Meta-Analyses (PRISMA) criteria. The stability of the knee joint is only marginally improved with the combined reconstruction of $A C L$ and $A L L$, and both reconstruction techniques show functional results. The main outcomes sought were patient function and graft stability and rupture rates after $A C L$ reconstruction. Out of the 421 studies identified, 6 were included in our meta-analysis. Study quality (internal validity) was assessed using the Cochrane risk-of-bias tool; in general, the studies included presented moderate-quality evidence. The graft rupture rate was higher in patients undergoing isolated ACL reconstruction (relative risk, 0.22 ; $95 \%$ confidence interval, 0.12 to $0.41 ; p<0.00001$ ).

\section{Introdução}

As rupturas do ligamento cruzado anterior (LCA) estão entre as lesões mais comuns no joelho, e o número de reconstruções do LCA aumentou nas últimas décadas, alcançando $\sim 130.000$ procedimentos realizados anualmente. ${ }^{1}$

Estudos mostraram que a incidência de reconstrução do LCA aumentou ao longo dos anos, particularmente em mulheres, bem como aquelas com $<20$ anos e aquelas com $\geq 40$ anos.Contudo, as pesquisas realizadas nos últimos anos, bem como as medidas para redução de custos, podem ser mais bem atendidas, visando prevenção e resultados positivos nesses grupos, porém os cirurgiões devem estar cientes de que o dano pode ocorrer a partir da reconstrução do LCA. $^{1}$

A reconstrução do LCA visa restaurar a função e a estabilidade do joelho; entretanto, a estabilidade rotacional pode não ser completamente restaurada pelo uso da reconstrução padrão isolada. ${ }^{2}$

Reconstrução do LCA é um dos procedimentos mais comuns em cirurgia ortopédica. No entanto, mesmo com avanços nas técnicas e implantes cirúrgicos, alguns pacientes ainda apresentam frouxidão residual rotatória anterolateral após a reconstrução. ${ }^{3}$

Embora estudos individuais não tenham mostrado a superioridade da reconstrução combinada do LCA em comparação com a reconstrução isolada em termos de função e estabilidade, os princípios biomecânicos sugerem que uma abordagem combinada pode ser útil, portanto, agrupar (meta-análise) os estudos clínicos randomizados disponíveis pode ser esclarecedor.

Segundo o estudo de Saithna et al., ${ }^{4}$ a reconstrução combinada do LCA e do ligamento anterolateral (LLA) está associada a uma redução significativa nas taxas de ruptura do enxerto do LCA e a um índice muito baixo de complicações, entretanto registra-se um aumento no risco de reoperação.

A persistente instabilidade rotacional após a reconstrução padrão isolada do LCA tem sido amplamente descrita, e tem sido demonstrado que mantém correlação direta com piores resultados no pós-operatório. ${ }^{5}$ A lesão do ligamento anterolateral (LLA) tem mostrado um papel relevante na gênese da instabilidade rotacional do joelho. ${ }^{3,6}$

Muitas publicações anatômicas definiram o LLA como um ligamento distinto. ${ }^{6}$ Entretanto, alguns autores propuseram a associação da reconstrução do LCA e do LLA em indivíduos deficientes para aumentar ainda mais a estabilidade do joelho no pós-operatório. ${ }^{7}$

Depois de muitos anos de vigoroso debate na literatura, um painel de pesquisadores internacionais e clínicos que estão entre os mais influentes na cirurgia do LCA chegaram finalmente a um consenso: o LLA existe. ${ }^{8}$

O LLA foi descrito pela primeira vez em 1879 pelo Dr. Paul Segond como uma "faixa fibrosa perolada e resistente" que poderia resultar em uma fratura avulsão do planalto tibial quando o joelho fosse rotacionado internamente à força: a fratura deSegond (1879). 
No início do século XIX, os anatomistas franceses Vallois e depois Josttiveramum interesse nas estruturas anterolaterais do joelho. Depois disso, não foi até 1976, quando Hughston et al. descreveram um "terço médio do ligamento capsular lateral," que o interesse nas estruturas anterolaterais do joelho foirenovado. ${ }^{9,10}$ Numerosos estudos se seguiram e o LLA foi denominado de várias maneiras diferentes, resultando em alta confusão em torno da anatomia anterolateral do joelho. ${ }^{11}$

A falta de consenso ainda predomina entre os especialistas do LCA em relação à confiabilidade da reconstrução combinada, considerando a controvérsia que envolve tanto a anatomia da LLA quanto a biomecânica. ${ }^{12,13}$ Ensaios clínicos com alto nível de evidência e acompanhamento a longo prazo podem ser úteis para determinar a confiabilidade do procedimento combinado no cenário clínico.

Desta forma, o objetivo da presente pesquisa é comparar, por meio de uma metanálise, os resultados da reconstrução combinada LCA e do (LLA), comparado com a reconstrução isolada padrão, em pacientes com lesão crônica do LCA.

A hipótese estabelecida no presenteestudo é de que os pacientes que foram submetidos à reconstrução combinada do LCA e do LLA exibem menor frouxidão residual e melhores desfechos clínicos se comparados com a reconstrução isolada.

\section{Metodologia}

Para identificar ensaios clínicos randomizados (ECR) comparando a reconstrução combinada do LCA (reconstrução com-

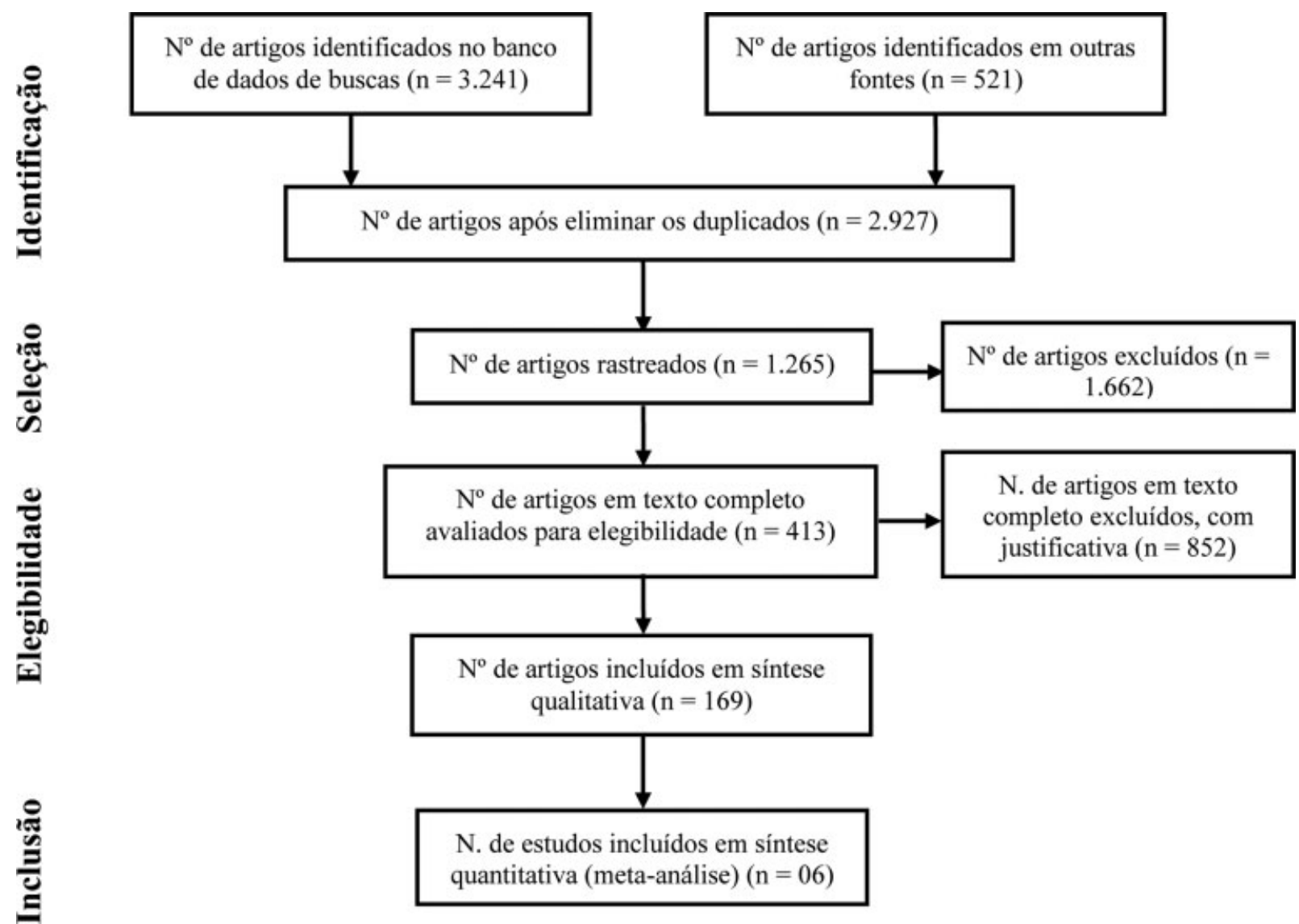

binada) com a reconstrução isolada do LCA, foram pesquisados artigos nas bases MEDLINE, EMBASE, SPORTDiscus, LILACS e Cochrane Central RegisterofControlledTrials, e foram seguidosos critérios de Itens de Relatórios Preferidos para Revisões Sistemáticas e Metanálises (PRISMA) ( - Figura 1).

Dois autores pesquisaram de forma independente as bases de dados eletrônicas entre abril e junho de 2019. Ensaios em andamento e recentemente concluídos foram identificados por pesquisano ClinicalTrials.gov. Não aplicamos nenhuma restrição com base em idiomas e os serviços de tradução foram usados quando necessário. Listas de referência de artigos relevantes foram verificadas quanto à sua completude. Também trabalhamos com resumos de conferências (disponíveison-line entre o período de 2010 a2019) da Sociedade Internacional de Artroscopia, Cirurgia do Joelho e Medicina Esportiva Ortopédica; Sociedade Ortopédica Americana de Medicina Esportiva; e a American AcademyofOrthopaedicSurgeons.

Nossa pesquisa bibliográfica identificou um total de 421 estudos, e após a exclusão de relatos obviamente irrelevantes e duplicados, 10artigos foram recuperados para avaliação. Nós reconhecemos dois relatórios do mesmo estudo; embora tenhamos combinado dados úteis de ambos os relatórios, a informação mais relevante foi extraída do relatório de texto completo. ${ }^{8}$ Nós excluímos outro estudo que não era um ECR. Os seis estudos restantes preencheram os critérios de inclusão para nossa revisão sistemática. ${ }^{3}$

A qualidade do estudo (validade interna) foi avaliada usando a ferramenta Cochrane risco-de-viés. ${ }^{11}$ Em geral, 
encontramos os estudos incluídos como sendo de qualidade moderada nessa escala.

Todas as análises estatísticas foram realizadas usando o software estatístico Review Manager 5.3 (RevMan 5.3; Centro Nórdico Cochrane, The Cochrane Collaboration, Copenhague, Dinamarca).${ }^{14}$ Os efeitos do tratamento foram expressos como razões de risco (RRs) para resultados dicotômicos e diferenças médias para resultados contínuos em intervalos de confiança de 95\% (IC95\%). Uma meta-análise de efeito fixo foi realizada para dados considerados homogêneos. A heterogeneidade dos efeitos do tratamento foi avaliada visualmente pela observação de ICs sobre parcelas florestais, além de sua direção e magnitude. Além disso, $\mathrm{I}^{2}$ estatísticas foram calculadas para uma avaliação objetiva da heterogeneidade.

A análise de subgrupos foi realizada quando possível. 0 viés de publicação foi avaliado por inspeção visual de gráficos de funil a partir de resultados primários. As avaliações podem não ser precisas devido ao pequeno número de tentativas. Os estudos estão bem distribuídos por todo o enredo e o viés de publicação não é uma grande preocupação. Como medida de segurança, pesquisamos a literatura cinzenta sobre esse assunto para encontrar trabalhos com resultados não relevantes ou negativos.

Os principais resultados que procuramos foram função do paciente, estabilidade e rupturas do enxerto após a reconstrução do LCA. Dos 421 estudos identificados, 6 estudos foram incluídos ( $n=776$ participantes; seguimento, 12-84 meses; homens-mulheres na razão de 2,17:1) em nossa meta-análise.

Nenhum dos estudos forneceu evidências de nível 1. Dois dos estudos alcançaram nível de evidência 2, com randomização. Os demais apresentaram nível de evidência 3 e 4 .

\section{Resultados}

Dos seis artigos selecionados, todos apresentaram seguimento de técnicas de reconstrução do LCA e do LAL. Todos os estudos juntos incluíram 776 pacientes, 402 foram submetidos a reconstrução do LCA e do LLA. Um artigo comparou a reconstrução do LCA isolada com a reconstrução do LCA e do LLA. Outro estudo comparou três técnicas de reconstrução do LCA: reconstrução com banda única, reconstrução com banda dupla e reconstrução anatômica com banda única associada à reconstrução do LLA. Um terceiro estudo também comparou três técnicas anatômicas de reconstrução do LCA: com enxerto do tendão patelar, enxerto quádruplo de tendões flexores e enxerto de tendões flexores combinado com reconstrução do LLA.

Todas as análises estatísticas foram realizadas utilizando o software estatístico Review Manager 5.3(The Nordic Cochrane Center, The Cochrane Collaboration, Copenhagen, Dinamarca). Os efeitos do tratamento foram expressos como razões de risco (RRs) para desfechos dicotômicos e diferenças médias para desfechos contínuos com IC95\%. Para os dados julgados homogêneos, uma meta-análise de efeito fixo foi realizada. A heterogeneidade dos efeitos do tratamento foi avaliada visualmente pela observação de ICs sobrepostos em parcelas no forestplot e a direção e magnitude dos efeitos do tratamento. Além disso, I estatísticas foram calculadas para uma avaliação objetiva da heterogeneidade. Alta heterogeneidade foi indicada pela ausência de sobreposição de ICs em parcelas no forestplot e I $>50 \%$ e as razões para heterogeneidade foram investigadas. Análise de subgrupo foi realizada quando viável.

Os gráficos de funil mostram o erro padrão (SE) e a razão de risco (RR) para os casos de ruptura do enxerto. O viés de publicação foi avaliado por inspeção visual de gráficos de funil a partir de desfechos primários (-Figura 2). As avaliações podem não ter precisão devido ao pequeno número de tentativas. Os estudos estão bem distribuídos ao longo da trama e o viés de publicação não é uma grande preocupação. Como medida de segurança, pesquisamos literatura cinza na área com o objetivo de encontrar estudos com resultados não relevantes ou negativos.

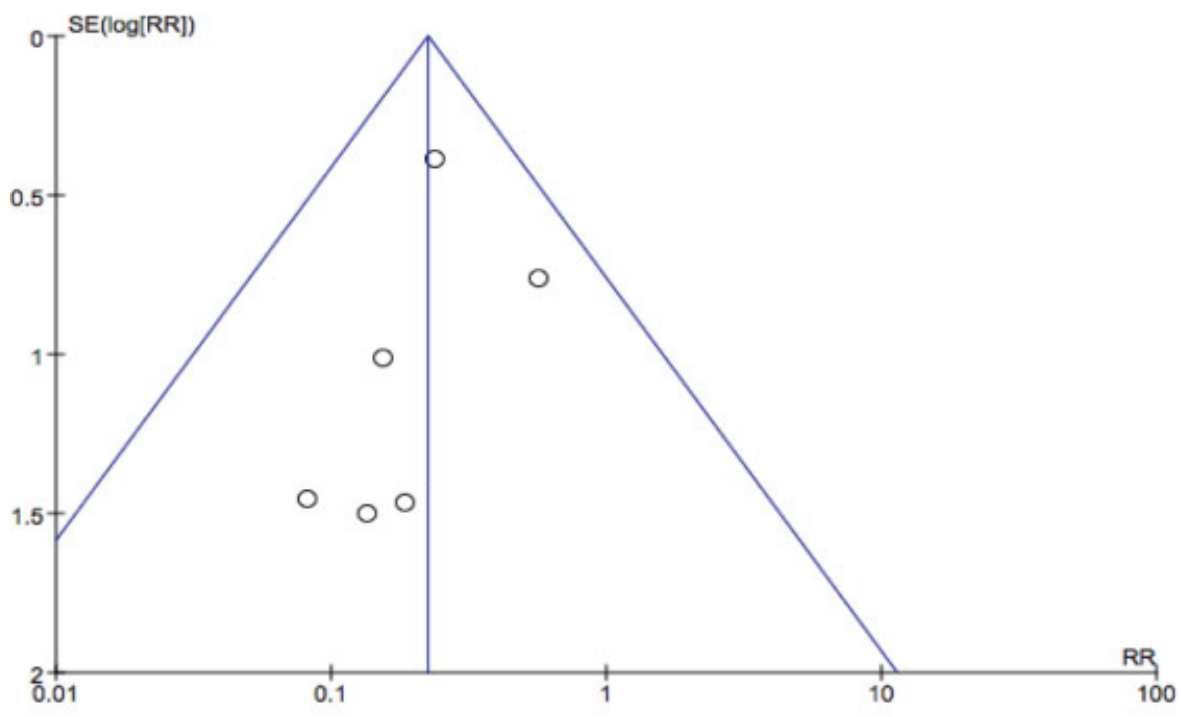

Fonte: Elaboração própria dos autores (2019).

Fig. 2 Gráfico de funil mostrando o erro padrão (SE) e a razão de risco (RR) para os casos de ruptura do enxerto. 
28 Reconstrução combinada do LCA e LLA comparada á reconstrução isolada do LCA Bucar et al.

\begin{tabular}{|c|c|c|c|c|c|c|c|c|c|c|}
\hline \multirow[b]{2}{*}{ Study or Subgroup } & \multicolumn{2}{|c|}{ LCA e LLA (combinada) } & \multicolumn{2}{|c|}{ LCA (isolada) } & \multicolumn{2}{|r|}{ Risk Ratio } & \multirow{2}{*}{\multicolumn{3}{|c|}{$\begin{array}{c}\text { Risk Ratio } \\
\text { M.H, Flxed, } 95 \% \mathrm{Cl}\end{array}$}} & \\
\hline & Events & Total & Events & Total & Weight & M-H, Flxed, $95 \% \mathrm{Cl}$ Year & & & & \\
\hline Kim et al. & 2 & 23 & 7 & 46 & $9.1 \%$ & $0.57[0.13,2.53] 2012$ & & & + & \\
\hline lbrahim et al. & 0 & 53 & 3 & 50 & $7.0 \%$ & $0.13[0.01,2.55] 2017$ & & & & \\
\hline Sonnery-Cottet et al. & 9 & 221 & 18 & 105 & $47.6 \%$ & $0.24[0.11,0.51] 2017$ & & - & & \\
\hline Helito et al. & 0 & 33 & 5 & 68 & $7.1 \%$ & $0.18[0.01,3.24] 2018$ & & & & \\
\hline Camilo et al. & 1 & 30 & 13 & 60 & $16.9 \%$ & $0.15[0.02,1.12] 2018$ & & & & \\
\hline Lee et al. & 0 & 42 & 6 & 45 & $12.3 \%$ & $0.08[0.00,1.42] 2019$ & & & & \\
\hline Total $(95 \% \mathrm{Cl})$ & & 402 & & 374 & $100.0 \%$ & $0.22[0.12,0.41]$ & & & & \\
\hline Total events & 12 & & 52 & & & & & & & \\
\hline $\begin{array}{l}\text { Heterogeneity: Chi }= \\
\text { Test for overall effect: }\end{array}$ & $\begin{array}{l}2.29, d f=5(P= \\
Z=4.82(P<0.0\end{array}$ & $p^{2}=0 \%$ & & & & & 0.001 & $\begin{array}{c}0.1 \\
\text { LCA E LLA }\end{array}$ & ${ }^{1}{ }_{\text {LCA }}{ }^{10}$ & 1000 \\
\hline
\end{tabular}

Fonte: Elaboração própria dos autores (2019).

Fig. 3 Gráfico de Forest Plot resultante da metanálisepara os casos de ruptura do enxerto.

A partir da comparação dos casos de ruptura do enxerto (-Figura 3), o gráfico de forestplot mostra diferença significativa $(p<0,05)$ no número de casos que foram submetidos apenas à reconstrução isolada do LCA e aqueles que foram submetidos à reconstrução combinada do LCA e do LLA, de forma que a taxa de ruptura de enxerto é significativamente maior no grupo de pacientes submetidos á reconstrução isolada do LCA (RR 0,22; IC95\%: 0,12-0,41; $p<0,00001$ ).

A diferença média lado a lado (e desvio padrão) na translação anterior da tíbia, medida com artrômetro KT2000 diferiu significativamente $(p<0.05)$ entre os pacientes submetidos à reconstrução isolada do LCA, comparados aos pacientes submetidos à reconstrução combinada do LCA (RR -0.65; IC95\%: -0.78--0.51; $p<0,00001)$. Deste modo, a média da translação anterior da tíbia é significativamente menor entre os pacientes submetidos à reconstrução combinada do LCA.

Em síntese, na avaliação da frouxidão ligamentar com uso do artrômetro KT-2000, observa-se que o LCA combinado com o LLA permite menor translação anterior da tíbia do que a reconstrução isolada do ACL (-Figura 4).

Os escores médios do teste Lysholm não diferiram significativamente $(p>0,05)$ entre os pacientes submetidos à reconstrução isolada do LCA, comparados aos pacientes submetidos à reconstrução combinada do LCA (RR 1,11; IC95\%: $-0,20-2,42 ; p=0,10$ ).

Os escores médios subjetivos do IKDC não diferiram significativamente $(p>0,05)$ entre os pacientes submetidos à reconstrução isolada do LCA, comparados aos pacientes submetidos à reconstrução combinada do LCA (RR 1,05; IC95\%: -0,47-2,56; $p=0,17$ ).

\section{Discussão}

O principal achado da presentemetanálisefoi que, comparada apenas à reconstrução isolada do LCA, a reconstrução combinada do LCA e do LLA não resultou em diferenças significativas na função do joelho.

Resultados relativamente consistentes da reconstrução isolada do LCA foram relatados e os resultados mostraram recuperação da função do joelho na maioria dos pacientes. No entanto, a estabilidade rotacional pode não ser restaurada apenas pela reconstrução isolada. 0 principal achado da nossa metanálise foi que, comparada apenas à reconstrução isolada do LCA, a reconstrução combinada LCA e LLA não resultou em diferenças significativas na função do joelho. Embora a estabilidade do joelho tenha sido superior no grupo de reconstrução

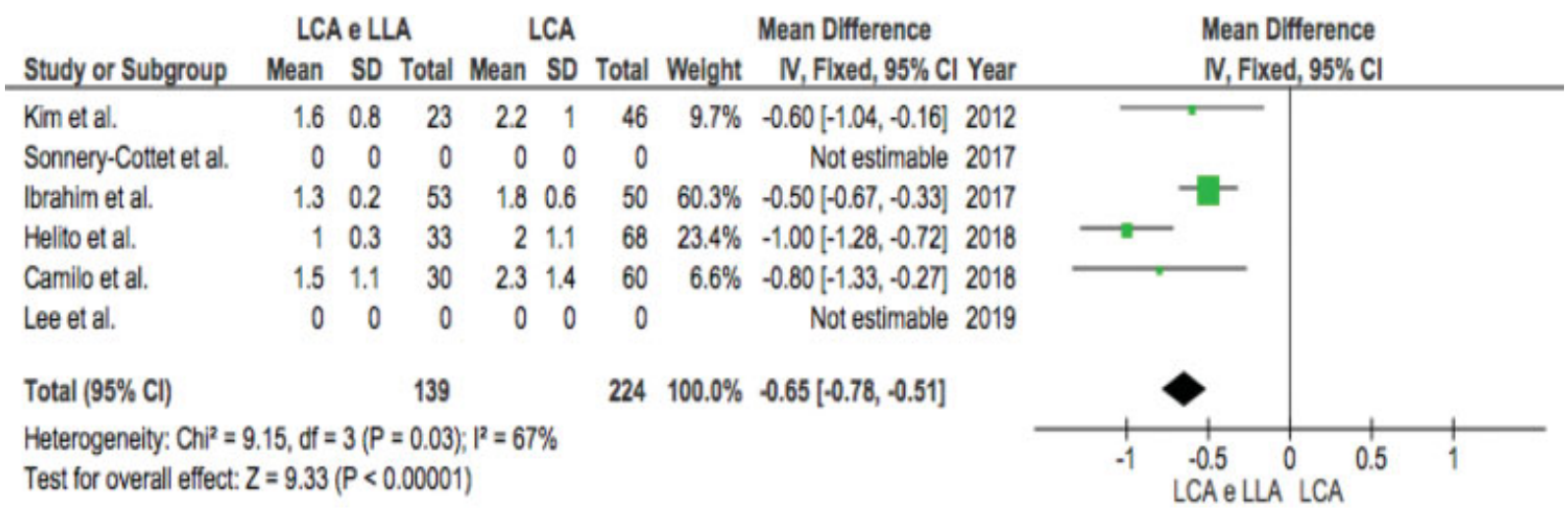

Fonte: Elaboração própria dos autores (2019).

Fig. 4 Gráfico de Forest Plot resultante da Metanálise para comparações dos resultados do teste KT-2000 com artrômetro após a reconstrução do LCA. 
combinada do LCA, os resultados do IKDC e do teste Lysholm foram apenas marginalmente melhorados.

Com base na avaliação da frouxidão ligamentar com o uso do artrômetro KT-2000, observamos que o LCA combinado com o LLA permite menor translação anterior do que a reconstrução isolada do LCA. Além disso, uma melhora na taxa de falha do enxerto após LCA combinada também foi relatada.

A pesquisa de Helito et al. ${ }^{3}$ apontou evidências de que a reconstrução combinada do LCA e do LLA em pacientes com lesão crônica do LCA é uma solução eficaz e segura e leva a bons resultados funcionais sem aumento da taxa de complicações. A relevância clínica desse achado foi a possibilidade de indicar esse tipo de procedimento quando os pacientes apresentam $>12$ meses após a lesão cirúrgica. ${ }^{3}$

Resultados semelhantes também foram apontados no estudo realizado por Saithna et al., onde os resultados clínicos da reconstrução articular avançada do LCA demonstraram uma redução significativa nas correntes de ruptura do LCA e taxas melhoradas de retorno aos esportes em comparação com a reconstrução isolada do LCA. ${ }^{4}$

Esse achado é apoiado por estudos laboratoriais que demonstraram que a associação de reconstrução do LCA e os sintomas extra-articulatórios laterais são procedimentos que protegem o acúmulo de cargas doLCA e é a recuperação mais confiável da cinemática normal do joelho em comparação com a reconstrução isolada do LCA.

Uma melhora na taxa de falha do enxerto após RLCA + RLLA combinada também foi relatada por Helito et al. ${ }^{3}$ Em sua coorte de pacientes com um acompanhamento mínimo de 2anos, a taxa de falha do enxerto foi de 0 e 7,3\% em pacientes com LTA + RTA e LCA, respectivamente $(p>0,05)$.

Uma outra pesquisa realizada por Sonnery-Cottet et al. mostrou que uma grande série comparativa prospectiva de 502 reconstruções do LCA realizadas com uma de 3técnicas cirúrgicas diferentes e um acompanhamento mínimo de 2anos demonstrou taxas significativamente reduzidas de ruptura do enxerto do LCA em uma população de alto risco (pacientes jovens participando de esportes) após reconstrução combinada do LCA e do LLA em comparação com uma coorte pareada submetida a reconstrução isolada do LCA. ${ }^{11}$

Inderhaug et al. mostraram que apesar das inúmeras descrições técnicas dos procedimentos anterolaterais, o conhecimento é limitado quanto ao efeito do ângulo de flexão do joelho durante a fixação do enxerto. ${ }^{15}$

Buscando determinar o efeito do ângulo de flexão do joelho durante a fixação do enxerto na cinemática da articulação tibiofemoral para uma reconstrução modificada da tenodeseLemaire ou do LLA combinada com a reconstrução do LCA, Inderhaug et al. mostraram que no LCA combinado e na deficiência anterolateral, a reconstrução isolada do LCA deixou frouxidão residual tanto na translação anterior como na rotação interna. ${ }^{15} \mathrm{~A}$ tradução anterior foi restaurada para todas as combinações de procedimentos LCA e anterolateral. A reconstrução combinada do LCA e o procedimento de LLA restauraram a cinemática intacta do joelho quando o enxerto foi fixado em extensão completa, mas quando o enxerto foi fixado em $30^{\circ}$ e $60^{\circ}$, o procedimento combinado deixou frouxidão residual na rotação interna $(p=0,043)$. A recons- trução combinada do LCA e o procedimento modificado de Lemaire restauraram a rotação interna, independentemente do ângulo de flexão do joelho na fixação do enxerto. Quando a reconstrução combinada do LCA e os estados do procedimento lateral foram comparados com o estado reconstruído apenas do LCA, uma redução significativa na frouxidão da rotação interna foi observada com a tenodese modificada de Lemaire, mas não com o procedimento LLA.

Em síntese, o estudo biomecânico dos autores citados acima demonstraram que, quando existe uma lesão combinada do LCA e anterolateral, um LCA-L isolado não consegue restaurar a estabilidade normal do joelho. ${ }^{15}$

Com base na avaliação da frouxidão ligamentar com o uso do artrômetro KT-2000, Kim et al. observaram que a reconstrução combinada do LCA e do canto posterolateral permitiu menor translação anterior do que a reconstrução isolada do LCA. ${ }^{5}$ No entanto, não conseguiu identificar diferenças significativas entre os dois grupos em termos de resultados funcionais.

Ibrahim et al. mostraram que a reconstrução combinada do LCA e do LLA mostrou-se eficaz para melhorar os resultados subjetivos e objetivos. ${ }^{16}$ No entanto, esses achados não foram significativamente superiores à reconstrução isolada do LCA, exceto para os resultados dos testes de lassidão do joelho. Isso pode indicar que a reconstrução doLLA não deve ser realizada rotineiramente para pacientes submetidos à reconstrução do LCA.

\section{Considerações Finais}

A estabilidade articular do joelho melhorou apenas marginalmente com reconstrução combinada do LCA e do LLA, e o resultado funcional foi semelhante para as duas técnicas de reconstrução estudadas.

A reconstrução do LCA em combinação com a reconstrução doLLA reduz significativamente a frouxidão ligamentar com o uso do artrômetro KT-2000 e mostrou uma menor taxa de ruptura do enxerto do que a reconstrução LCA isolada, embora não tenham sido apontadas diferenças significativas nos resultados do teste funcional entre os dois grupos.

A quantidade reduzida de artigos selecionados para compor a metanálisee a qualidade moderada dos mesmos estão entre as principais limitações da presentepesquisa.

\section{Conflito de Interesses}

Os autores declaram não haver conflito de interesses.

\section{Referências}

1 Mall NA, Chalmers PN, Moric M, et al. Incidence and trends of anterior cruciate ligament reconstruction in the United States. Am J Sports Med 2014;42(10):2363-2370

2 Rezende FC. Reconstrução combinada intra e extra-articular versus reconstrução intra-articular isolada do ligamento cruzado anterior: revisão sistem [tese]. São Paulo: Universidade Federal de São Paulo (UNIFESP); 2016

3 Helito CP, Camargo DB, Sobrado MF, et al. Combined reconstruction of the anterolateral ligament in chronic ACL injuries leads to better clinical outcomes than isolated ACL reconstruction. Knee Surg Sports Traumatol Arthrosc 2018;26(12):3652-3659 
30 Reconstrução combinada do LCA e LLA comparada á reconstrução isolada do LCA Bucar et al.

4 Saithna A, Thaunat M, Delaloye JR, Ouanezar H, Fayard JM, SonneryCottet B. Sonnery-CottetB. Combined ACL and anterolateral ligament reconstruction. JBJS Essential Surg Tech 2018;8(01):e2

5 Kim SJ, Choi DH, Hwang BY. The influence of posterolateral rotatory instability on ACL reconstruction: comparison between isolated ACL reconstruction and ACL reconstruction combined with posterolateral corner reconstruction. J Bone Joint Surg Am 2012;94(03):253-259

6 Claes S, Vereecke E, Maes M, Victor J, Verdonk P, Bellemans J. Anatomy of the anterolateral ligament of the knee. J Anat 2013; 223(04):321-328

7 Dodds AL, Halewood C, Gupte CM, Williams A, Amis AA. The anterolateral ligament: Anatomy, length changes and association with the Segond fracture. Bone Joint J 2014;96-B(03):325-331

8 Tulloch S, Getgood A. Consideration of lateral augmentation in anatomic anterior cruciate ligament reconstruction. Ann Joint 2019;4:15

9 Hughston JC, Andrews JR, Cross MJ, Moschi A. Classification of knee ligament instabilities. Part II. The lateral compartment. J Bone Joint Surg Am 1976;58(02):173-179

10 Cavaignac E, Faruch M, Wytrykowski K, et al. Ultrasonographic Evaluation of Anterolateral Ligament Injuries: Correlation With Magnetic Resonance Imaging and Pivot-Shift Testing. Arthroscopy 2017;33(07):1384-1390
11 Sonnery-Cottet B, Saithna A, Cavalier M, et al. Anterolateral ligament reconstruction is associated with significantly reduced ACL graft rupture rates at a minimum follow-up of 2 years: a prospective comparative study of 502 patients from the SANTI study group. Am J Sports Med 2017;45(07):1547-1557

12 Guenther D, Rahnemai-Azar AA, Bell KM, et al. The anterolateral capsule of the knee behaves like a sheet of fibrous tissue. Am J Sports Med 2017;45(04):849-855

13 Kittl C, El-Daou H, Athwal KK, et al. The role of the anterolateral structures and the ACL in controlling laxity of the intact and ACLdeficient knee. Am J Sports Med 2016;44(02):345-354

14 Geeslin AG, Moatshe G, Chahla J, et al. Anterolateral Knee Extra-articular Stabilizers: A Robotic Study Comparing Anterolateral Ligament Reconstruction and Modified Lemaire Lateral Extra-articular Tenodesis. Am J Sports Med 2018;46(03): 607-616

15 Inderhaug E, Stephen JM, Williams A, Amis AA. Anterolateral tenodesis or anterolateral ligament complex reconstruction: effect of flexion angle at graft fixation when combined with ACL reconstruction. Am J Sports Med 2017;45(13):3089-3097

16 Ibrahim SA, Shohdy EM, Marwan Y, et al. Anatomic reconstruction of the anterior cruciate ligament of the knee with or without reconstruction of the anterolateral ligament: a randomized clinical trial. Am J Sports Med 2017;45(07):1558-1566 